Research Paper

\title{
Decrease in Plasma Cyclophilin A Concentration at 1 Month after Myocardial Infarction Predicts Better Left Ventricular Performance and Synchronicity at 6 Months: A Pilot Study in Patients with ST Elevation Myocardial Infarction
}

\author{
Ching-Hui Huang ${ }^{1,2^{*}}$, Chia-Chu Chang ${ }^{3,4^{*}}$, Chen-Ling Kuo ${ }^{5}$, Ching-Shan Huang5, Chih-Sheng Lin ${ }^{2^{\bowtie}}$, \\ Chin-San Liu $5,6,7 \bowtie$ \\ 1. Division of Cardiology, Department of Internal Medicine, Changhua Christian Hospital, Changhua 500, Taiwan \\ 2. Department of Biological Science and Technology, National Chiao Tung University, Hsinchu 300, Taiwan \\ 3. Division of Nephrology, Department of Internal Medicine, Changhua Christian Hospital, Changhua 500, Taiwan \\ 4. School of Medicine, Chung Shan Medical University, Taichung 404, Taiwan \\ 5. Vascular and Genomic Research Center, Changhua Christian Hospital, Changhua 500, Taiwan \\ 6. Department of Neurology, Changhua Christian Hospital, Changhua 500, Taiwan \\ 7. Graduate Institute of Integrative Medicine, China Medical University, Taichung 404, Taiwan \\ * These authors contributed equally to the manuscript.
}

$\triangle$ Corresponding authors: Chin-San Liu, MD, PhD. Department of Neurology, Vascular and Genomic Research Center, Changhua Christian Hospital, 135 Nanhsiao Street Changhua 500, Taiwan. Tel: +886-4-7238595 ext.4752 E-mail: liu48111@gmail.com Chih-Sheng Lin, PhD. Department of Biological Science and Technology, National Chiao Tung University, 75 Po-Ai Street, Hsinchu 300, Taiwan. Tel.: +886-3-5131338 E-mail: lincs@mail.nctu.edu.tw.

(c) Ivyspring International Publisher. This is an open-access article distributed under the terms of the Creative Commons License (http://creativecommons.org/ licenses/by-nc-nd/3.0/). Reproduction is permitted for personal, noncommercial use, provided that the article is in whole, unmodified, and properly cited.

Received: 2014.08.05; Accepted: 2014.11.05; Published: 2015.01.01

\begin{abstract}
Background: Cyclophilin A (CyPA) concentration increases in acute coronary syndrome. In an animal model of acute myocardial infarction, administration of angiotensin-converting-enzyme inhibitor was associated with lower left ventricular (LV) CyPA concentration and improved LV performance. This study investigated the relationships between changes in plasma CyPA concentrations and LV remodeling in patients with ST-elevation myocardial infarction (STEMI).

Methods and Results: We enrolled 55 patients who underwent percutaneous coronary intervention for acute STEMI. Plasma CyPA, matrix metalloproteinase (MMP), interleukin-6 and high-sensitivity C-reactive protein concentrations were measured at baseline and at one-month follow-up. Echocardiography was performed at baseline and at one-, three-, and six-month follow-up. Patients with a decrease in baseline CyPA concentration at one-month follow-up $(n=28)$ had a significant increase in LV ejection fraction (LVEF) (from $60.2 \pm 11.5 \%$ to $64.6 \pm 9.9 \%, p<0$. $001)$ and preserved LV synchrony at six months. Patients without a decrease in CyPA concentration at one month $(n=27)$ did not show improvement in LVEF and had a significantly increased systolic dyssynchrony index (SDI) (from $1.170 \pm 0.510 \%$ to $1.637 \pm 1.299 \%, p=0.042$ ) at six months. Multiple linear regression analysis showed a significant association between one-month CyPA concentration and six-month LVEF. The one-month MMP-2 concentration was positively correlated with one-month CyPA concentration and LV SDI.

Conclusions: Decreased CyPA concentration at one-month follow-up after STEMI was associated with better LVEF and SDI at six months. Changes in CyPA, therefore, may be a prognosticator of patient outcome.
\end{abstract}


Key words: Acute myocardial infarction; Cyclophilin A; Left ventricular ejection fraction; Left ventricular dyssynchrony; Matrix metalloproteinase.

\section{Introduction}

Cyclophilin A (CyPA) is a ubiquitously distributed intracellular protein of the cyclophilin family that functions as an intracellular chaperone protein with peptidyl cis-trans prolyl-isomerase activity, and is the high-affinity receptor for the immunosuppressive drug cyclosporine [1]. During inflammatory processes and in response to oxidative stress, CyPA is released into the extracellular space by both inflamed living cells and dying cells [2-4] to provide protection against oxidative stress-induced apoptosis [5, 6]. However, extracellular CyPA is a potent chemoattractant for human monocytes and neutrophils, and enhanced expression of CyPA is involved in various pathophysiological processes such as atherosclerosis, myocardial ischemia-reperfusion injury, and aortic aneurysm formation [7-11]. CyPA is also an important mediator of angiotensin II-induced cardiac hypertrophy [12]. In addition, CyPA or its receptor extracellular matrix metalloproteinase inducer (EMMPRIN) has been shown to be associated with myocardial inflammation, ischemia, and remodeling [13]. The CyPA-EMMPRIN activation pathway may play a role in increasing the vulnerability of atherosclerotic plaques [14]. Patients with acute coronary syndrome have high plasma concentrations of CyPA, and $\mathrm{CyPA}$ is strongly expressed in the athermanous plaques of patients with acute myocardial infarction (AMI) [15]. In a rabbit model of AMI, CyPA concentration was up-regulated in the left ventricular (LV) wall, and administration of the angiotensin-converting-enzyme inhibitor (ACEI) ramipril caused an increase in antioxidant enzyme activities, resulting in a significant decrease in LV CyPA concentration, significant improvement in LVEF, and a marked reduction in LV remodeling [16]. Furthermore, disruption of the CyPA-EMMPRIN pathway was shown to reduce infarct size and preserve systolic function after myocardial ischemia and reperfusion in an animal model [17]. These results suggest that persistently high CyPA concentration in cardiac tissue after AMI may be a biomarker of LV remodeling and impaired systolic function. Plasma CyPA is secreted from monocytes and vascular wall cells in response to oxidative stress and inflammation, but can also be secreted by or leaked from damaged cardiomyocytes and interstitial fibroblasts [13]. Persistently elevated plasma circulating CyPA levels in patients after STEMI may reflect a state of increased oxidative stress and proinflammatory status. However, it is unknown whether persistent elevation of plasma CyPA con- centration after AMI affects LV performance.

Ischemia-reperfusion injury is characterized by an extensive inflammatory response with leukocyte recruitment, induction of a complex inflammatory chemokine network, and generation of matrix metalloproteinases (MMPs) [18]. Optimal healing requires a balanced MMP-mediated extracellular matrix turnover [19], and inadequate MMP activity triggers an intensified remodeling process leading to fibrosis and cardiac rupture [20, 21]. Experimental and clinical evidence indicates that MMP activity can modulate and predict remodeling [22, 23]. CyPA and its receptor EMMPRIN represent a ligand/receptor pair that critically regulates both leukocyte recruitment and MMP activity [3, 24, 25]. LV dyssynchrony is also an important predictor of LV remodeling after AMI [26], and is correlated with infarct size and LV function [27]. However, no previous studies have evaluated the relationship between plasma CyPA and MMP concentrations in terms of LV remodeling after AMI, or the associations of these concentrations with LV dyssynchrony.

This study was conducted to test the hypothesis that persistent high plasma CyPA concentration after AMI results in LV remodeling. This study investigated the relationships between plasma CyPA concentrations, LVEF and LV dyssynchrony in patients with STEMI. The relationships between plasma MMP concentrations and CyPA concentrations, LVEF, and LV dyssynchrony were also evaluated.

\section{Materials and Methods}

\section{Subjects and study protocol}

This single center study prospectively enrolled 55 consecutive patients who underwent primary percutaneous coronary intervention (PCI) for de novo acute STEMI at the Changhua Christian Hospital, Taiwan between January 2010 and January 2011. We also enrolled a control group of 55 healthy age- and sex-matched volunteers to compare changes in plasma CyPA levels after STEMI. All eligible patients aged between 18 and 80 years who consented to participate in the study were included. Patients presenting with STEMI were eligible for primary PCI if the time from symptom onset to presentation was $<12$ hours. STEMI was diagnosed according to the universal definition of myocardial infarction [28]. Specifically, STEMI was diagnosed if all of the following were present: symptoms of cardiac ischemia, ST segment elevation of $>0.2 \mathrm{mV}$ in $\geq 2$ contiguous electro- 
cardiogram leads, and serum troponin I or creatine kinase-MB (CK-MB) levels in excess of the $9^{\text {th }}$ percentile reference limit within 24 hours of the onset of pain. The culprit vessel was identified based on clinical, electrocardiographic, and angiographic findings. Aspirin and clopidogrel were administered to all patients before PCI. The goal for door-to-balloon time (D2B time) was $<90$ minutes.

Baseline creatine phosphokinase, creatinine, hemoglobin, and hemoglobin A1c levels were routinely measured prior to PCI. Baseline lipid and glucose levels were measured after an 8-hour fast. CK-MB and troponin I levels were measured every 4 hours until they started to decline, and the peak values were recorded.

After PCI, patients received standard care, including administration of aspirin, clopidogrel, beta-blockers, ACEIs or angiotensin receptor blockers (ARBs) as appropriate. Patients also received smoking cessation and lifestyle counseling.

The study protocol was approved by the Institutional Review Board of the Changhua Christian Hospital, Taiwan. All subjects gave written informed consent for inclusion in the study.

\section{Measurement of plasma CyPA, MMP-2, MMP-9, interleukin (IL)-6, and high-sensitivity C-reactive protein (hsCRP) concentrations}

Plasma CyPA, MMP-2 and MMP-9 concentrations were obtained prior to PCI and at day 3 , day 7 and 1 month after the acute event. Plasma IL-6 and hsCRP concentrations were measured at baseline (prior to PCI) and at one-month follow-up. Plasma CyPA concentrations were measured using enzyme-linked immunosorbent assay kits from Wuhan USCN Science (Wuhan, China) in accordance with the manufacturer's instructions. Plasma MMP-2, MMP-9, IL-6, and hsCRP concentrations were measured by enzyme-linked immunosorbent assay kits from R\&D Systems (Minneapolis, MN, USA) in accordance with the manufacturer's instructions.

\section{Echocardiographic examinations}

Echocardiography was performed at baseline (within 2 days after PCI) and at one-, three-, and six-month follow-up. All 2-D echocardiographic parameters were measured according to the guidelines of the American Society of Echocardiography [29]. We used a modified Simpson's method to calculate LVEF as described by the American Echocardiographic Society [30]. Regional wall motion score index (WMSI) was calculated as the sum of wall motion scores divided by the number of visualized segments (from 17-segment model), where 1 indicates normal; 2, hypokinesis; 3, akinesis; and 4, dyskinesis [31]. Re- al-time 3-D echocardiographic images were obtained using an iE33 xMATRIX echocardiography system (Philips Medical Systems, Andover, MA, USA). The systolic dyssynchrony index (SDI) was defined as the standard deviation of the time-to-minimum systolic volume of $16 \mathrm{LV}$ segments expressed as a percentage of the R-R duration, as described by Kapetanakis et al. [32]. A higher index indicates greater LV dyssynchrony.

\section{Angiographic assessments}

All quantitative coronary angiographic measurements were performed by a single cardiologist who was blinded to the other investigation findings. The Thrombolysis in Myocardial Infarction (TIMI) flow [33] and myocardial blush grade [34] immediately after PCI were retrospectively reviewed by the same cardiologist, and the TIMI risk score was determined. Coronary artery lesions were classified according to the guidelines of the American College of Cardiologists/American Heart Association [35]. Repeat coronary angiographies were performed 6 months later to detect residual stenosis of the infarct-related artery.

\section{Statistical analysis}

Statistical analyses were performed using the SPSS statistical software package (version 15.0; Chicago, IL, USA). Values are presented as mean \pm SD. Differences between baseline CyPA levels and those measured at one-month follow-up were compared between patients with and those without a decrease in CyPA concentration using the Student's t-test. Changes in echocardiographic findings and MMP-2 and MMP-9 concentrations over time were compared between patients with and those without a decrease in CyPA concentration at 1 month using the paired-samples t-test. Changes in baseline SDI, IL-6, MMP-2, and MMP-9 concentrations at one-month were compared between patients with low and those with high baseline CypA concentration (below and above the median value, respectively) using the Student's t-test. The relationship between quartiles of 1-month CyPA concentration and 1-month MMP-2 concentration was analyzed using the Jonckheere-Terpstra test. Multiple linear regression analyses were performed to identify factors associated with 6-month LVEF, baseline SDI, and 6-month SDI. The analysis for 6-month LVEF included the baseline and 1-month CyPA concentrations and known prognostic factors for LVEF (peak CK-MB level, infarct-related artery, wall motion score index, and hsCRP concentrations). Further multiple regression analyses were performed using the same model, but replacing CyPA concentration with IL-6, MMP-2, or 
MMP-9 concentration as one of the covariates, to investigate the relationships between these variables and 6-month LVEF. The analyses for SDI included CyPA and MMP-2 concentrations and factors associated with LV dyssynchrony (age, sex, peak CK-MB level, IL-6 concentration, and LVEF). Receiver operator characteristic (ROC) curves were constructed to assess the predictive accuracy of CyPA for LVEF and MMP-2 for SDI. The area under the curves (AUC) for predicting LVEF with CyPA and SDI with MMP-2 were calculated. Correlations between MMP-2 concentrations and SDI were analyzed using Spearman's rho coefficient. A $p<0.05$ was regarded as statistically significant. As there were few data available regarding CyPA concentrations after AMI, we were unable to estimate sample size and study power.

\section{Results}

\section{Comparisons between patients with and without a decrease in plasma CyPA concen- tration between baseline and 1 month}

Plasma CyPA concentration in the AMI group was significantly higher than that in the control group $(61.3 \pm 24.2$ vs. $54.3 \pm 9.4 \mathrm{ng} / \mathrm{mL}, p=0.042)$. The characteristics of patients with $(\mathrm{n}=28)$ and without $(\mathrm{n}=$ 27) a decrease in CyPA concentration between baseline and 1 month are shown in Table 1. Changes in plasma CyPA concentrations over time in patients with and without a decrease in CyPA are shown in Figure 1. The 1-month CyPA concentration was significantly lower in patients with a decrease in CyPA concentration at 1 month than in patients without a decrease in CyPA concentration at 1 month (55.4 \pm $25.7 \mu \mathrm{g} / \mathrm{dL}$ vs. $71.2 \pm 26.0 \mu \mathrm{g} / \mathrm{dL}, p=0.021)$. No other significant differences were found between the two groups, including use of ACEIs and ARBs after PCI. However, plasma MMP-2 concentrations were significantly higher among patients without a decrease in CyPA concentration at 1 month $(45.3 \pm 41.7 \mathrm{ng} / \mathrm{mL}$ vs. $15.9 \pm 56.6 \mathrm{ng} / \mathrm{mL}, p=0.027)$.

Repeat PCI for restenosis of the infarct-related artery was performed in 9 patients with a decrease in CyPA concentration at 1 month and in 9 patients without a decrease in CyPA concentration at 1 month. There was no significant difference in infarct-related artery restenosis rate between the two groups $(p=$ 0.782). There were no deaths during the 6-month follow-up period.
Table 1. Comparisons between patients with and without a decrease in plasma CyPA concentration at 1 month.

\begin{tabular}{|c|c|c|c|}
\hline & $\begin{array}{l}\text { Decrease in } \\
\text { CyPA at } 1 \text { month } \\
(\mathrm{n}=28)\end{array}$ & $\begin{array}{l}\text { No decrease in } \\
\text { CyPA at } 1 \text { month } \\
(\mathrm{n}=27)\end{array}$ & $p$-value \\
\hline \multicolumn{4}{|l|}{ CyPA (ng/mL) } \\
\hline baseline & $65.7 \pm 25.7$ & $56.3 \pm 24.1$ & 0.150 \\
\hline 1 month & $55.4 \pm 25.7$ & $71.2 \pm 26.0$ & $0.021^{*}$ \\
\hline$\triangle \mathrm{CyPA}$ & $-10.4 \pm 12.7$ & $14.9 \pm 14.6$ & $<0.001^{* *}$ \\
\hline Sex (male/female) & $23 / 5$ & $24 / 3$ & 0.337 \\
\hline Age (years) & $57.7 \pm 12.3$ & $57.9 \pm 11.2$ & 0.960 \\
\hline Body mass index $\left(\mathrm{kg} / \mathrm{m}^{2}\right)$ & $25.6 \pm 3.4$ & $25.7 \pm 3.8$ & 0.894 \\
\hline $\begin{array}{l}\text { Creatine phosphokinase } \\
(\mathrm{u} / \mathrm{L})\end{array}$ & $1,959 \pm 1,774$ & $2,742 \pm 2,225$ & 0.139 \\
\hline CK-MB, peak (ng/mL) & $208 \pm 190$ & $265 \pm 196$ & 0.254 \\
\hline Troponin I, peak (ng/mL) & $7.02 \pm 21.01$ & $2.90 \pm 4.51$ & 0.362 \\
\hline Creatinine (mg/dL) & $0.95 \pm 0.21$ & $1.05 \pm 0.41$ & 0.257 \\
\hline WMSI & $1.27 \pm 0.21$ & $1.28 \pm 0.19$ & 0.949 \\
\hline Statin used (\%) & 84 & 83 & 0.910 \\
\hline TIMI risk score & $2.90 \pm 1.17$ & $2.69 \pm 1.10$ & 0.508 \\
\hline LVMI (g/m²) & $109 \pm 22$ & $110 \pm 28$ & 0.928 \\
\hline LVEF (\%) & $60.2 \pm 11.5$ & $61.2 \pm 10.1$ & 0.723 \\
\hline SDI (\%) & $1.50 \pm 1.28$ & $1.17 \pm 0.51$ & 0.214 \\
\hline EDV (mL) & $74.8 \pm 24.5$ & $77.1 \pm 17.8$ & 0.696 \\
\hline Hemoglobin (g/dL) & $14.7 \pm 1.6$ & $14.1 \pm 1.6$ & 0.159 \\
\hline Hematocrit (\%) & $57.1 \pm 15.0$ & $40.8 \pm 4.6$ & 0.292 \\
\hline Fasting glucose (mg/dL) & $153 \pm 80$ & $152 \pm 91$ & 0.950 \\
\hline Hemoglobin A1c (\%) & $6.5 \pm 1.7$ & $6.3 \pm 1.5$ & 0.706 \\
\hline Cholesterol (mg/dL) & $191 \pm 46$ & $191 \pm 51$ & 0.995 \\
\hline HDL-C (mg/dL) & $40.9 \pm 11.0$ & $41.9 \pm 9.4$ & 0.730 \\
\hline LDL-C (mg/dL) & $137 \pm 39$ & $132 \pm 41$ & 0.674 \\
\hline Culprit lesion & $\begin{array}{l}\mathrm{LAD}(16), \\
\mathrm{LCX}(3), \operatorname{RCA}(9)\end{array}$ & $\begin{array}{l}\operatorname{LAD}(14), \\
\operatorname{LCX}(3), \operatorname{RCA}(10)\end{array}$ & 0.718 \\
\hline D2B time (min) & $85.5 \pm 38.4$ & $84.3 \pm 38.0$ & 0.916 \\
\hline Myocardial blush grade & $2.10 \pm 0.87$ & $2.00 \pm 0.93$ & 0.693 \\
\hline Restenosis (n) & 9 & 9 & 0.782 \\
\hline ACEI use after PCI (\%) & 55 & 53 & 0.436 \\
\hline ARB use $\mathrm{PCI}(\%)$ & 31 & 24 & 0.163 \\
\hline \multicolumn{4}{|l|}{ hsCRP (mg/L) } \\
\hline baseline & $0.47 \pm 0.86$ & $0.47 \pm 0.44$ & 0.966 \\
\hline 1 month & $0.32 \pm 0.92$ & $0.46 \pm 0.76$ & 0.541 \\
\hline$\triangle \mathrm{hsCRP}$ & $-0.18 \pm 0.96$ & $-0.00 \pm 0.64$ & 0.431 \\
\hline \multicolumn{4}{|l|}{ IL-6 (pg/mL) } \\
\hline baseline & $17.1 \pm 16.0$ & $18.1 \pm 9.3$ & 0.761 \\
\hline 1 month & $10.8 \pm 8.5$ & $11.9 \pm 7.8$ & 0.651 \\
\hline$\Delta \mathrm{IL}-6$ & $-6.6 \pm 11.0$ & $-6.3 \pm 8.1$ & 0.190 \\
\hline \multicolumn{4}{|l|}{ MMP-2 (ng/mL) } \\
\hline baseline & $239 \pm 61$ & $212 \pm 54$ & 0.071 \\
\hline 1 month & $255 \pm 49$ & $257 \pm 43$ & 0.878 \\
\hline$\triangle \mathrm{MMP}-2$ & $15.9 \pm 56.6$ & $45.3 \pm 41.7$ & $0.027^{*}$ \\
\hline \multicolumn{4}{|l|}{ MMP-9 (ng/mL) } \\
\hline baseline & $203 \pm 181$ & $274 \pm 249$ & 0.213 \\
\hline 1 month & $54.3 \pm 52.4$ & $52.3 \pm 28.5$ & 0.856 \\
\hline$\Delta$ MMP-9 & $-149 \pm 160$ & $-221 \pm 242$ & 0.180 \\
\hline
\end{tabular}

Data are presented as mean \pm SD. All values are baseline values unless otherwise indicated. $\Delta$ indicates value changes between 1 month and baseline. Normal laboratory values of creatine- kinase-MB (CK-MB) range from $0.6-6.3 \mathrm{ng} / \mathrm{ml}$; Normal troponin-I concentration levels are $<0.04 \mathrm{ng} / \mathrm{mL}$.

${ }^{*} p<0.05$, Student's t-test.

ACEI: angiotensin-converting-enzyme inhibitor; ARB: angiotensin receptor blocker; CK-MB: creatine kinase-MB; CyPA: cyclophilin A; D2B: door-to-balloon; EDV: left ventricular end-diastolic volume; HDL-C: high-density lipoprotein cholesterol; hsCRP: high-sensitivity C-reactive protein; IL-6: interleukin 6; LAD: left anterior descending artery; LCX: left circumflex artery; LDL-C: low-density lipoprotein cholesterol; LVEF: left ventricular ejection fraction; LVMI: left ventricular mass index; MMP: matrix metalloproteinase; PCI: percutaneous coronary intervention; RCA: right coronary artery; SDI: systolic dyssynchrony index; TIMI: Thrombolysis in Myocardial Infarction; WMSI: wall motion score index. 


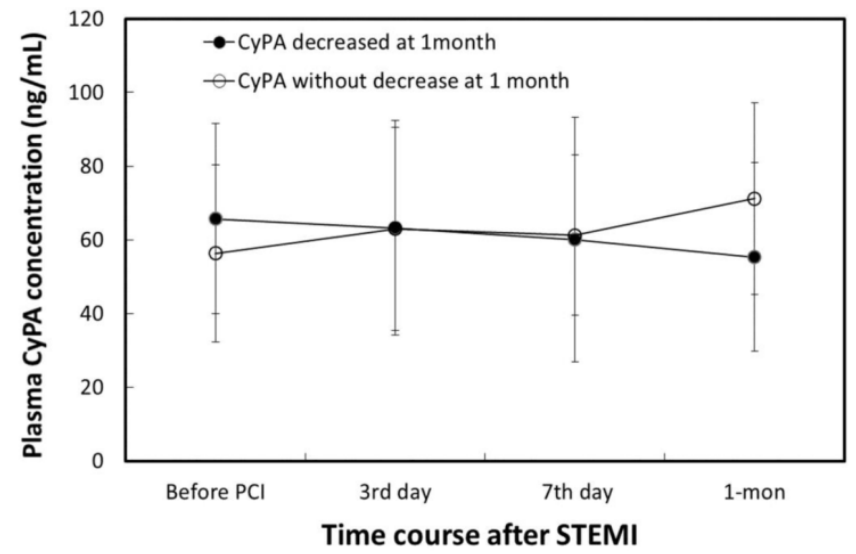

Figure 1. Changes in plasma CyPA concentration in patients with a decrease and without a decrease in plasma CyPA levels between baseline and 1 month. Group A comprised patients with a decrease in plasma CyPA concentration at 1 month. Group B comprised patients without a decrease in plasma CyPA concentration at 1 month. Al vs. A4, $(p<0.001)$. B1 vs. B4 $(p<0.001)$ (paired-samples t-test). A4 vs. B4 ( $p=0.021$, Student's t-test).

\section{Changes in echocardiographic findings and MMP concentrations over time in patients with and without a decrease in CyPA concen- tration at 1 month}

In patients with a decrease in CyPA concentration at 1 month, LVEF significantly improved at 1, 3 and 6 months $(p=0.006, p=0.018$, and $p=0.004$, respectively); however, in patients without a decrease in CyPA concentration at 1 month, there were no significant changes at any of the time points (Table 2). The wall motion score index improved significantly at all time points in both groups of patients (both $p<0.001$ ). SDI did not change significantly at 1,3 and 6 months in patients with a decrease in CyPA concentration at 1 month; however, in patients without a decrease in CyPA concentration at 1 month, SDI but deteriorated significantly at 3 months and at 6 months $(p=0.018$ at 3 months and 0.042 at 6 months). Patients without a decrease in CyPA concentration at 1 month also had a significant increase in MMP-2 concentration at 1 month $(p<0.001)$. Both groups of patients had a significant decrease in MMP-9 concentration at 1 month (both $p<0.001$ ).

\section{Comparisons between patients with low and high baseline CyPA concentrations}

The median baseline CyPA concentration was 60 $\mathrm{ng} / \mathrm{mL}$. Patients with a low baseline CyPA concentration (below the median) had a significantly lower baseline SDI than patients with a high baseline CyPA concentration (above the median) $(p=0.043$, Table 3 ). Patients with a low baseline CyPA concentration also had significantly lower 1-month IL-6 and MMP-2 concentrations than patients with a high baseline
CyPA concentration $(p=0.019$ and $p=0.007$, respectively).

\section{Relationship between quartiles of 1-month CyPA concentration and 1-month MMP-2 concentration}

Patients were divided into 4 groups according to quartiles of 1-month CyPA concentration $(\leq 39.7$, $39.8-59.7,59.8-73.1$, and $\geq 73.2 \mathrm{ng} / \mathrm{mL}$ ). Trend analysis showed that 1-month MMP-2 concentration was positively correlated with quartiles of 1-month CyPA concentration $(p=0.026)$ (Figure 2).

Table 2. Changes in echocardiographic findings and MMP concentrations over time in patients with and without a decrease in CyPA concentration at 1 month.

\begin{tabular}{|c|c|c|}
\hline AMI patients & Measured value & $p$-value \\
\hline \multicolumn{3}{|c|}{ With decrease in CyPA concentration at 1 month $(n=28)$} \\
\hline \multicolumn{3}{|l|}{ LVEF (\%) } \\
\hline baseline & $60.2 \pm 11.5$ & \\
\hline 1 month & $64.3 \pm 10.2$ & $0.006^{* *}$ \\
\hline 3 months & $63.5 \pm 9.1$ & $0.018^{*}$ \\
\hline 6 months & $64.6 \pm 9.9$ & $0.004^{* *}$ \\
\hline \multicolumn{3}{|l|}{ SDI (\%) } \\
\hline baseline & $1.50 \pm 1.28$ & \\
\hline 1 month & $1.69 \pm 1.22$ & 0.323 \\
\hline 3 months & $1.50 \pm 0.77$ & 0.999 \\
\hline 6 months & $1.74 \pm 1.67$ & 0.099 \\
\hline \multicolumn{3}{|c|}{ MMP-2 (ng/mL) } \\
\hline baseline & $239 \pm 61$ & \\
\hline 1 month & $255 \pm 49$ & 0.129 \\
\hline \multicolumn{3}{|c|}{ MMP-9 (ng/mL) } \\
\hline baseline & $204 \pm 181$ & \\
\hline 1 month & $54 \pm 52$ & $<0.001^{* * *}$ \\
\hline \multicolumn{3}{|l|}{ WMSI } \\
\hline baseline & $1.27 \pm 0.21$ & \\
\hline 6 months & $1.14 \pm 0.18$ & $<0.001^{\text {** }}$ \\
\hline \multicolumn{3}{|c|}{ Without decrease in CyPA concentration at 1 month $(n=27)$} \\
\hline \multicolumn{3}{|c|}{ LVEF (\%) } \\
\hline baseline & $61.2 \pm 10.2$ & \\
\hline 1 month & $62.5 \pm 12.1$ & 0.292 \\
\hline 3 months & $61.5 \pm 13.5$ & 0.830 \\
\hline 6 months & $60.6 \pm 12.9$ & 0.701 \\
\hline \multicolumn{3}{|l|}{ SDI (\%) } \\
\hline baseline & $1.17 \pm 0.51$ & \\
\hline 1 month & $1.64 \pm 1.35$ & 0.075 \\
\hline 3 months & $1.81 \pm 1.26$ & $0.018^{*}$ \\
\hline 6 months & $1.64 \pm 1.30$ & $0.042^{*}$ \\
\hline \multicolumn{3}{|c|}{ MMP-2 (ng/mL) } \\
\hline baseline & $212 \pm 54$ & \\
\hline 1 month & $257 \pm 43$ & $<0.001^{* * *}$ \\
\hline \multicolumn{3}{|c|}{ MMP-9 (ng/mL) } \\
\hline baseline & $274 \pm 249$ & \\
\hline 1 month & $52 \pm 29$ & $<0.001^{* *}$ \\
\hline \multicolumn{3}{|l|}{ WMSI } \\
\hline baseline & $1.28 \pm 0.19$ & \\
\hline 6 months & $1.17 \pm 0.23$ & $<0.001^{* * *}$ \\
\hline
\end{tabular}


Table 3. Comparisons between patients with low and high baseline CyPA concentrations.

\begin{tabular}{llll}
\hline & $\begin{array}{l}\text { Low baseline CyPA }(< \\
60 \mathrm{ng} / \mathrm{mL} ; \mathrm{n}=28)\end{array}$ & $\begin{array}{l}\text { High baseline CyPA } \\
(\geq 60 \mathrm{ng} / \mathrm{mL} ; \mathrm{n}=27)\end{array}$ & $p$-value \\
\hline $\begin{array}{l}\text { SDI, baseline (\%) } \\
\text { IL-6 (pg/mL) }\end{array}$ & $1.07 \pm 0.43$ & $1.62 \pm 1.29$ & $0.043^{*}$ \\
baseline & $15.8 \pm 11.4$ & $19.9 \pm 14.7$ & 0.207 \\
$\begin{array}{l}1 \text { month } \\
\text { MMP-2 (ng/mL) }\end{array}$ & $9.0 \pm 6.0$ & $15.2 \pm 9.5$ & $0.019^{*}$ \\
baseline & $216 \pm 53$ & $235 \pm 61$ & 0.193 \\
$\begin{array}{l}\text { month } \\
\text { MMP-9 (ng/mL) }\end{array}$ & $240 \pm 45$ & $271 \pm 42$ & $0.007^{* *}$ \\
baseline & $253 \pm 226$ & $22 \pm 195$ & 0.556 \\
1 month & $49.2 \pm 34.9$ & $57.1 \pm 48.3$ & 0.469 \\
\hline
\end{tabular}

Data are presented as mean \pm SD.

${ }^{*} p<0.05,{ }^{* *} p<0.01$, Student's t-test.

CyPA: cyclophilin A; IL-6: interleukin-6; MMPs: matrix metalloproteinase; SDI: systolic dyssynchrony index.

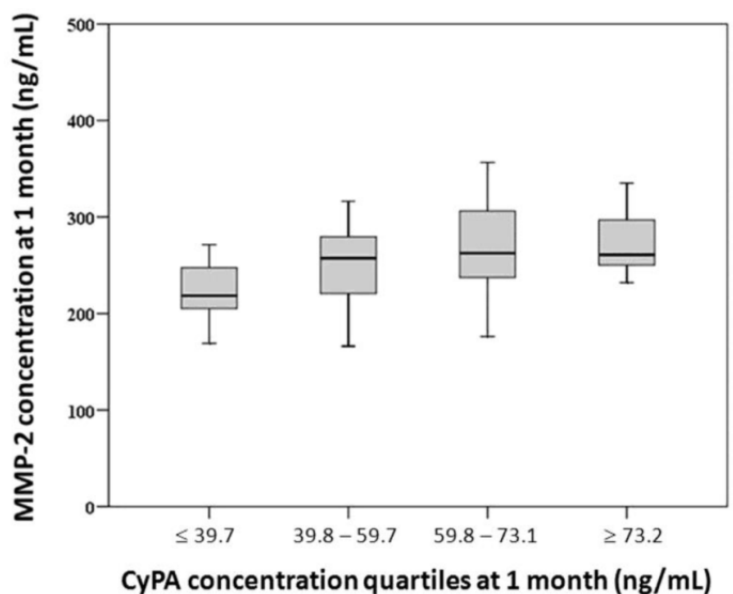

Figure 2. Relationship between quartiles of 1-month cyclophilin A (CyPA) concentration and 1-month matrix metalloproteinase-2 (MMP-2) concentration. Trend analysis showed that 1-month MMP-2 concentration was positively correlated with quartiles of 1-month CyPA concentration (Jonckheere-Terpstra test, $p=0.026$ ).

\section{Factors associated with 6-month LVEF}

Multivariate analysis revealed that 6-month LVEF was independently associated with 1-month CyPA concentration and baseline wall motion score index, but not with baseline CyPA concentration, baseline or 1-month hsCRP concentration, infarct location, or peak CK-MB level (Table 4). Further analyses using the same model but with IL-6, MMP-2, or MMP-9 concentration as variables rather than CyPA concentration did not show significant associations between these variables and 6-month LVEF.

\section{Evaluation of plasma CyPA at 1 month as a predictor of LVEF at $\mathbf{6}$ months}

The median LVEF at 6 months was $64 \%$. Using this as a reference value, patients with STEMI were divided into two subgroups, namely a high 6-month LVEF group and a low 6-month LVEF group. The dependent variable was 6-month LVEF. The area under the curves (AUC) for plasma 1-month CyPA con- centration as a predictor of 6-month LVEF was 0.734 (95\% CI, 0.618-0.869, $p=0.001$ ) (Figure 3).

\section{Factors associated with baseline and 6-month SDI}

Multivariate analyses revealed that baseline SDI was independently associated with baseline MMP-2 concentration and baseline LVEF and that 6-month SDI was independently associated with 1-month MMP-2 concentration and 6-month LVEF (Table 5). Baseline and 6-month SDI were significantly higher in females than in males.

\section{Evaluation of plasma MMP-2 concentration at 1 month as a predictor of SDI at 6 months}

The median SDI at 6-month follow-up was $1.42 \%$. Using this as a reference value, patients with STEMI were divided into two subgroups, namely a high 6-month SDI group and a low 6-month SDI group. SDI at 6 months was considered as a dependent variable. The AUC for plasma 1-month MMP-2 concentration as a predictor of 6-month SDI was 0.682 (95\% CI, 0.547-0.817, $p=0.015$ ) (Figure 4).

Table 4. Results of multiple regression analysis with 6-month LVEF as the dependent variable.

\begin{tabular}{lllll}
\hline Predictors & $\begin{array}{l}\text { Regression } \\
\text { coefficient }\end{array}$ & $\begin{array}{l}\text { Standard } \\
\text { error }\end{array}$ & $\begin{array}{l}\text { Standardized } \\
\text { coefficient }\end{array}$ & P-value \\
\hline Intercept & 120.329 & 9.850 & & $<0.001^{\text {*** }}$ \\
CyPA, baseline & 0.080 & 0.078 & 0.164 & 0.310 \\
CyPA, 1 month & -0.160 & 0.069 & -0.356 & $0.026^{*}$ \\
CK-MB, peak & -0.006 & 0.007 & -0.093 & 0.417 \\
IRA & -0.201 & 1.394 & -0.016 & 0.886 \\
WMSI & -41.263 & 6.997 & -0.692 & $<0.001^{\text {*** }}$ \\
hsCRP, baseline & 1.174 & 1.808 & 0.071 & 0.519 \\
hsCRP, 1 month & 2.786 & 1.689 & 0.180 & 0.106
\end{tabular}

$\mathrm{R}^{2}=0.618$. ${ }^{*} p<0.05,{ }^{* * *} p<0.001$.

CK-MB: creatine kinase-MB; CyPA: cyclophilin A; hsCRP: high-sensitivity C-reactive protein; IRA: infarct-related artery; LEVF: left ventricular ejection fraction; WMSI: wall motion score index.

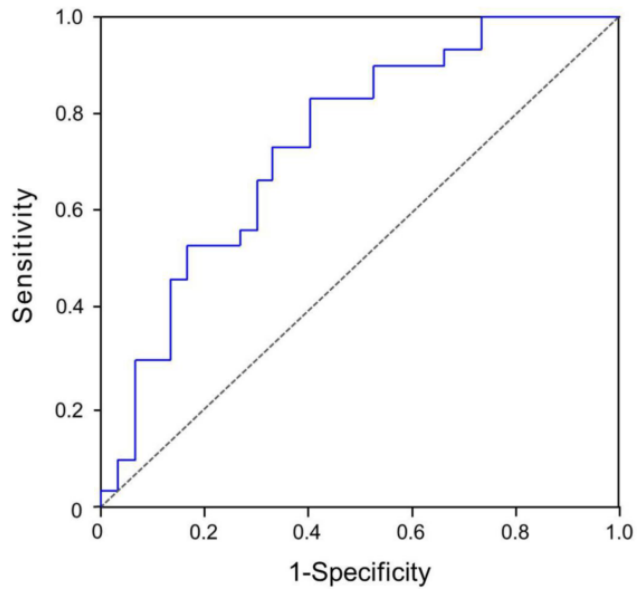

Figure 3. Evaluation of plasma CyPA concentration at 1 month as a predictor of 6-month LVEF in STEMI patients after primary balloon angioplasty. The area under the curves (AUC) for plasma 1 -month CyPA concentration as a predictor of 6-month LVEF was $0.734(95 \% \mathrm{Cl}, 0.618-0.869, p=0.001)$. 


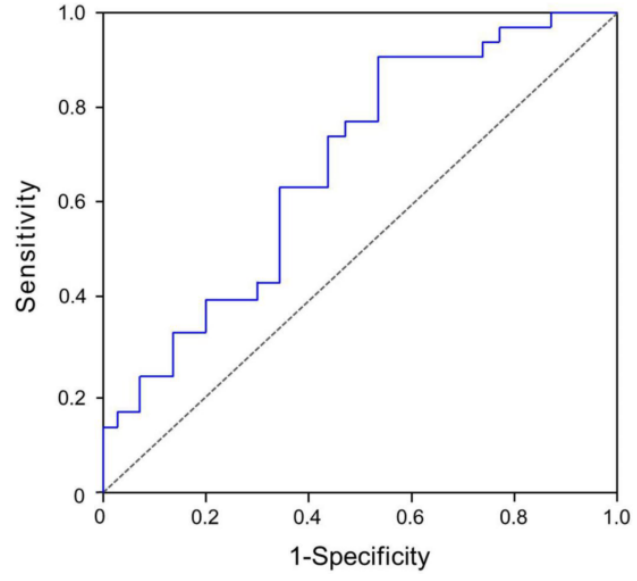

Figure 4. Evaluation of plasma MMP-2 concentration at 1 month as a predictor of 6-month SDI in STEMI patients after primary balloon angioplasty. The area under the curves (AUC) for plasma 1-month MMP-2 concentration as a predictor of 6-month SDI were $0.682(95 \% \mathrm{Cl}, 0.547-0.817, p=0.015)$.

Table 5. Results of multiple regression analyses with baseline or 6-month SDI as the dependent variable.

\begin{tabular}{|c|c|c|c|c|}
\hline Predictors & $\begin{array}{l}\text { Regression } \\
\text { coefficient }\end{array}$ & $\begin{array}{l}\text { Standard } \\
\text { error }\end{array}$ & $\begin{array}{l}\text { Stand- } \\
\text { ardized } \\
\text { coeffi- } \\
\text { cient }\end{array}$ & P-value \\
\hline \multicolumn{5}{|c|}{ Baseline SDI $\left(R^{2}=0.616\right)$} \\
\hline Intercept & 3.284 & 1.076 & & $0.004^{* *}$ \\
\hline Age & 0.022 & 0.009 & 0.256 & $0.015^{*}$ \\
\hline Sex & -0.643 & 0.280 & -0.233 & $0.026^{*}$ \\
\hline CK-MB, peak & -0.001 & 0.001 & -0.183 & 0.069 \\
\hline CyPA, baseline & -0.006 & 0.004 & -0.142 & 0.192 \\
\hline MMP-2, baseline & 0.004 & 0.002 & 0.251 & $0.013^{*}$ \\
\hline IL-6, baseline & 0.004 & 0.007 & 0.061 & 0.552 \\
\hline LVEF, baseline & -0.051 & 0.009 & -0.560 & $<0.001^{* * *}$ \\
\hline \multicolumn{5}{|c|}{ 6-month SDI $\left(R^{2}=0.641\right)$} \\
\hline Intercept & 8.246 & 1.966 & & $<0.001^{* *}$ \\
\hline Age & -0.019 & 0.017 & -0.142 & 0.259 \\
\hline Sex & -2.232 & 0.495 & -0.487 & $<0.001^{* *}$ \\
\hline CK-MB, peak & -0.001 & 0.001 & -0.177 & 0.132 \\
\hline CyPA, 1 month & -0.013 & 0.009 & -0.181 & 0.135 \\
\hline MMP-2, 1 month & 0.009 & 0.004 & 0.248 & $0.034^{*}$ \\
\hline IL-6, 1 month & 0.038 & 0.021 & 0.195 & 0.082 \\
\hline LVEF, 6 months & -0.079 & 0.016 & -0.596 & $<0.001^{* *}$ \\
\hline
\end{tabular}

${ }^{*} p<0.05,{ }^{* *} p<0.01,{ }^{* * *} p<0.001$.

CK-MB: creatine kinase-MB; CyPA: cyclophilin A: IL-6: interleukin-6; LVEF: left ventricular ejection fraction; MMP-2: matrix metalloproteinase-2; SDI: systolic dyssynchrony index.

\section{Correlations between MMP-2 concentrations and SDI}

Baseline MMP-2 concentration was positively correlated with baseline, 1-month, and 6-month SDI. The 1-month MMP-2 concentration was positively correlated with 1-month and 6-month SDI (Table 6).

\section{Factors associated with changes in plasma CyPA concentration between baseline and 1 month}

Multivariate analyses revealed that $\triangle \mathrm{CyPA}$ (plasma CyPA concentration at 1 month minus base- line CyPA concentration) was positively associated with D2B time, peak CK-MB level and infarct location (Table 7).

Table 6. Correlations between MMP-2 concentrations and SDI.

\begin{tabular}{lllll}
\hline & $\begin{array}{c}\text { MMP-2 at } \\
\text { baseline }(\mathrm{n}= \\
55)\end{array}$ & P1 & $\begin{array}{c}\text { MMP-2 at 1 } \\
\text { month }(\mathrm{n}=55)\end{array}$ & \\
& \multicolumn{2}{c}{ P2 } & & \\
\hline SDI, baseline & 0.465 & $<0.001^{* * *}$ & & $0.011^{*}$ \\
SDI, 1 month & 0.360 & $0.007^{* *}$ & 0.342 & $0.015^{*}$ \\
SDI, 6 months & 0.370 & $0.005^{* *}$ & 0.325 & \\
\hline
\end{tabular}

Values are Spearman's rho coefficient.

${ }^{*} p<0.05,{ }^{* *} p<0.01,{ }^{* * *} p<0.001$.

P1 refers to the correlation between baseline MMP-2 concentration and SDI. P2 refers to the correlation between 1-month MMP-2 concentration and SDI.

MMP-2: matrix metalloproteinase-2; SDI: systolic dyssynchrony index.

Table 7. Results of linear regression analyses with $\triangle \mathrm{CyPA}$ as the dependent variable.

\begin{tabular}{lllll}
\hline Predictors & $\begin{array}{l}\text { Regression } \\
\text { coefficient }\end{array}$ & $\begin{array}{l}\text { Standard } \\
\text { error }\end{array}$ & $\begin{array}{l}\text { Standardized } \\
\text { coefficient }\end{array}$ & $p$-value \\
\hline Constant & -8.613 & 26.655 & & 0.760 \\
Age, year & -0.180 & 0.331 & -0.088 & 0.591 \\
Female vs. Male & 5.906 & 8.184 & 0.114 & 0.476 \\
CK-MB, peak & 0.043 & 0.020 & 0.380 & $0.042^{*}$ \\
Troponin I, peak & -0.125 & 0.197 & -0.101 & 0.530 \\
IRA-LAD vs. & -10.940 & 7.655 & -0.259 & 0.164 \\
IRA-RCA & & & & \\
IRA-LCX vs. & -37.768 & 12.515 & -0.483 & $0.005^{*}$ \\
IRA-RCA & & & & \\
D2B time & 0.239 & 0.082 & 0.436 & $0.007^{*}$ \\
IL-6 & -0.279 & 0.234 & -0.194 & 0.243
\end{tabular}

$\mathrm{R}^{2}=0.409 .{ }^{*} p<0.05$

$\triangle \mathrm{CyPA}$ indicated plasma CyPA concentration at 1 month minus CyPA concentration at baseline.

CK-MB: creatine kinase-MB; IRA: infarct-related artery; LAD: left anterior descending artery; LCX: left circumflex artery; RCA: right coronary artery.

\section{Discussion}

This study found that patients with a decrease in plasma CyPA concentration between baseline and 1 month after STEMI had better LV function at 6-month follow-up than patients without a decrease in CyPA concentration at 1-month follow-up. This finding suggests that prolonged inflammation may be associated with relatively poor recovery of $\mathrm{LV}$ function after STEMI. Multiple linear regression analyses identified only 1-month CyPA concentration and the baseline wall motion score index as predictive factors for 6-month LV function. Baseline IL-6, MMP-2, MMP-9, and hsCRP concentrations, infarct-related artery, and degree of myocardial injury were not significantly associated with 6-month LV function. This indicates that CyPA concentration may be a better predictor of LV function after STEMI than hsCRP concentration, which may be because CyPA is a marker of both oxidative stress and inflammation, whereas CRP is a marker of inflammation only [15]. 
Our findings support those of Piot et al. [36], who found that administration of cyclosporine to patients with STEMI at the time of reperfusion during PCI reduced the infarct size as estimated by myocardial enzyme levels. The same group of researchers analyzed cardiac MRI findings in 28 patients from the same study group [37]. At 6 months, patients who had received cyclosporine had a persistent reduction in infarct size compared with the control group. In a mini-pig model of irreversible anterior wall AMI, intra-coronary administration of cyclosporine inhibited the inflammatory cascade, reduced LV infarct size, and reduced LV remodeling [38]. The mechanisms underlying these outcomes are still unclear. Cyclosporine is a potent inhibitor of the mitochondrial permeability-transition pore, which plays an important role in attenuating ischemia-reperfusion injury [39]. Cyclosporine also binds to CyPA, thereby reducing circulating CyPA concentration. The results of these studies provide indirect evidence that it may be beneficial to decrease the CyPA concentration after AMI.

During AMI, reactive oxygen species (ROS) are generated in the ischemic myocardium, especially after reperfusion [18]. Although the mechanism is complex, basically inflammatory cytokines, e.g. IL-6, are produced in the ischemic region and surrounding myocardium as a host reaction [18], which in turn stimulate the production of ROS [42]. ROS and inflammatory cytokines then work in tandem to activate MMPs and collagen deposition, which contribute to LV remodeling after myocardial infarction [43, 44]. Additional evidence for the role of ROS play in AMI is provided by Chen et al and Wang et al, who showed that infarct size is markedly reduced in transgenic mice that overexpress the antioxidant protein superoxide dismutase (SOD) [40,41]. CyPA plays a crucial and synergistic role in ROS augmentation [45]. In addition, CyPA has been shown to activate NF- $\kappa \mathrm{B}$ and promote IL-6 release in monocytes [46] and is most likely broadly involved in the regulation of pro-inflammatory activity of monocytes/ macrophages.

The renin-angiotension-aldosterone system (RAAS) plays an important role in the pathophysiology of LV remodeling and progression of heart failure after myocardial infarction. It also known that RAAS is an ROS inducer that promotes CyPA secretion [45]. Pharmacologic inhibition of RAAS attenuates oxidative stress and LV remodeling and might decrease CyPA levels. There is a large body of clinical evidence that angiotensin-converting enzyme inhibitors and angiotensin receptor antagonists improve the prognosis of patients by attenuating the progression of LV remodeling $[47,48]$. In this study, there were no sig- nificant differences in the proportions of patients treated with ACEIs or ARBs between the groups with and without a decrease in CyPA concentration at 1 month. In a rabbit model of AMI, ACEI administration resulted in a decrease in CyPA concentration in the LV wall [16]. The discrepancy may be due to the relative small sample size of our study. Further studies are needed to clarify the difference between CyPA concentration in the LV and in plasma after ACEI administration.

The mechanism underlying the association between a decrease in CyPA concentration and preservation of LV function after AMI probably involves disruption of the CyPA-EMMPRIN pathway. Expression of CyPA and CD147 are increased in infarcted myocardium in both humans and mice [17]. In a mouse model of myocardial ischemia and reperfusion injury, Seizer et al found that CyPA/CD147 interaction was a critical mechanism in the pathophysiology of infarcted myocardium and mediated monocyte chemotaxis [17]. Disruption of the CyPA-EMMPRIN pathway resulted in a reduction in infarct size and preserved systolic function after myocardial ischemia and reperfusion, but anti-CD147 treatment did not result in further protection from ischemia-reperfusion injury in $\mathrm{CyPA}^{-/-}$mice. We hypothesize that a decrease in CyPA concentration after AMI reduces the ligand for CyPA/CD147 axis stimulation, thereby reducing chemotaxis and improving preservation of LV function.

LV dyssynchrony is defined as a difference in the timing of contractions among different segments of the LV. Dyssynchronous contraction results in increased wall stress in late contracting areas, increased myocardial oxygen consumption and a decrease in cardiac efficiency $[49,50]$. These in turn result in a vicious cycle of deteriorating cardiac function. Early recognition of dyssynchrony may enable physicians to predict a higher probability of LV remodeling after AMI [51]. In this study, SDI did not change significantly at 1-month, 3-month, or 6-month follow-up in patients with a decrease in CyPA concentration at 1 month, but deteriorated significantly in patients without a decrease in CyPA concentration at 1 month. LV systolic dyssynchrony has been shown to be a predictor of cardiac remodeling after AMI [26, 52], and has been shown to be independently associated with an unfavorable prognosis $[27,53]$. Patients in the highest quartile of 1-month CyPA concentration also had the highest 1-month MMP-2 concentrations (Figure 2). Furthermore, MMP-2 concentrations were significantly correlated with baseline, 1-month, and 6-month SDI (Table 5). Multiple linear regression analysis and ROC curve analysis (Figure 4) found that MMP-2 concentration was a significant predictor of 
SDI. These results suggest that patients without a decrease in CyPA concentration at 1 month have a higher 1-month MMP-2 concentration and higher 6-month SDI. To the best of our knowledge, this is the first study to examine the relationship between MMP-2 concentration and systolic dyssynchrony. A previous study found that higher MMP-2 concentration was an independent predictor of ventricular remodeling 6 months after AMI [23]. In addition, pharmacologic and genetic inhibitions of MMP were found to attenuate the development of heart failure in a rat model of AMI $[22,53]$. Nilsson et al [54] found that plasma MMP-2 concentration was positively correlated with final infarct size and LV dysfunction in patients with STEMI. In addition, LV systolic function and infarct size have been shown to be significantly associated with LV dyssynchrony $[27,55,56]$. In this study, a higher baseline CyPA concentration was significantly associated with a higher 1-month MMP-2 concentration, even though it was not associated with baseline MMP-2 concentration. This result may reflect augmentation of inflammation and generation of reactive oxygen species in patients with higher baseline CyPA concentrations. As CyPA plays a role in the inflammatory response and MMP production via the CyPA-EMMPRIN pathway, it is not surprising that MMP-2 concentrations were associated with LV systolic dyssynchrony (expressed as SDI) in this study.

The change in CyPA between 1 month and baseline was positively correlated with D2B time and cardiac muscle injury degree. This observation highlights the importance of early reperfusion. With decreased D2B time, STEMI patients are likely to have decreased plasma CyPA concentrations at 1 month. We found that female patients had more pronounced LV dyssynchrony than male patients. This difference in susceptibility may be related to differences in sex hormone levels and needs further investigation.

\section{Study limitations}

This study is limited by its relatively small sample size and short follow-up period. The number of female patients was small, and differences between males and females should be investigated further. Larger studies with longer follow-up are needed to confirm our findings.

\section{Conclusions}

Patients with a decrease in plasma CyPA concentration between baseline and 1 month after primary PCI for STEMI had better preservation of LV systolic function and better LV synchrony at 6 months. Patients with a higher baseline CyPA concentration had a significantly increased MMP-2 con- centration at 1 month after STEMI. The 1-month MMP-2 concentration was positively correlated with quartiles of 1-month CyPA concentration, and was significantly correlated with LV dyssynchrony. CyPA may be a novel biomarker for risk stratification of patients with STEMI, and clinical trials to examine the effects of drugs to decrease plasma CyPA concentrations are warranted.

\section{Acknowledgments}

This work was supported by Changhua Christian Hospital (research grant No. 100-CCH-IRP-08). We would like to thank Dr. Yu-Jun Chang, a consultant for the epidemiology and biostatistics center at the Changhua Christian Hospital.

\section{Competing Interests}

The authors have declared that no competing interest exists.

\section{References}

1. Handschumacher RE, Harding MW, Rice J, et al. Cyclophilin: a specific cytosolic binding protein for cyclosporin A. Science 1984; 226: 544-7.

2. Jin ZG, Melaragno MG, Liao DF, et al. Cyclophilin A is a secreted growth factor induced by oxidative stress. Circ Res 2000; 87: 789-96.

3. Arora K, Gwinn WM, Bower MA, et al. Extracellular cyclophilins contribute to the regulation of inflammatory responses. J Immunol 2005; 175: 517-22.

4. Galat A. Peptidylproline cis-trans-isomerases: immunophilins. Eur J Biochem 1993; 216: 689-707.

5. Seko $\mathrm{Y}$, Fujimura T, Taka $\mathrm{H}$, et al. Hypoxia followed by reoxygenation induces secretion of cyclophilin A from cultured rat cardiac myocytes. Biochem Biophys Res Commun 2004; 317: 162-8.

6. Lee J. Role of cyclophilin a during oncogenesis. Arch Pharm Res 2010; 33: 181-7.

7. Sherry B, Yarlett N, Strupp A, Cerami A. Identification of cyclophilin as a proinflammatory secretory product of lipopolysaccharide-activated macrophages. Proc Natl Acad Sci U S A 1992; 89: 3511-5.

8. Xu Q, Leiva M, Fischkoff $S$, et al. Leukocyte chemotactic activity of cyclophilin. J Biol Chem 1992; 267: 11968-71.

9. Satoh K, Nigro P, Matoba T, et al. Cyclophilin A enhances vascular oxidative stress and the development of angiotensin II-induced aortic aneurysms. Nat Med 2009; 15: 649-56.

10. Satoh K, Matoba T, Suzuki J, et al. Cyclophilin A mediates vascular remodeling by promoting inflammation and vascular smooth muscle cell proliferation. Circulation 2008; 117: 3088-98.

11. Nigro $\mathrm{P}$, Satoh $\mathrm{K}, \mathrm{O}^{\prime}$ Dell MR, et al. Cyclophilin A is an inflammatory mediator that promotes atherosclerosis in apolipoprotein E-deficient mice. J Exp Med 2011; 208: 53-66.

12. Satoh K, Nigro P, Zeidan A, et al. Cyclophilin A promotes cardiac hypertrophy in apolipoprotein E-deficient mice. Arterioscler Thromb Vasc Biol 2011; 31: 1116-23.

13. Hattori F. Extracellular cyclophilin A as a humoral factor modulating cardiovascular inflammatory responses. J Mol Cell Cardiol 2012; 53: 1-2.

14. Yan J, Zang X, Chen R, et al. The clinical implications of increased cyclophilin A levels in patients with acute coronary syndromes. Clin Chim Acta 2012; 413: 691-5.

15. Satoh K, Fukumoto Y, Sugimura K, et al. Plasma cyclophilin A is a novel biomarker for coronary artery disease. Circ J 2013; 77: 447-55.

16. Chen $\mathrm{CY}$, Lee BC, Hsu HC, et al. A proteomic study of the effects of ramipril on post-infarction left ventricular remodelling in the rabbit. Eur J Heart Fail 2008; 10: 740-8.

17. Seizer $P$, Ochmann $C$, Schönberger $T$, et al. Disrupting the EMMPRIN (CD147)-cyclophilin A interaction reduces infarct size and preserves systolic function after myocardial ischemia and reperfusion. Arterioscler Thromb Vasc Biol 2011; 31: 1377-86.

18. Hori M, Nishida K. Oxidative stress and left ventricular remodelling after myocardial infarction. Cardiovasc Res 2009; 81: 457-64.

19. Yarbrough WM, Mukherjee R, Escobar GP, et al. Selective targeting and timing of matrix metalloproteinase inhibition in post-myocardial infarction remodeling. Circulation 2003; 108: 1753-9.

20. Heymans S, Luttun A, Nuyens D, et al. Inhibition of plasminogen activators or matrix metalloproteinases prevents cardiac rupture but impairs therapeutic angiogenesis and causes cardiac failure. Nat Med 1999; 5: 1135-42. 
21. Matsumura S, Iwanaga S, Mochizuki S, et al. Targeted deletion or pharmacological inhibition of MMP-2 prevents cardiac rupture after myocardial infarction in mice. J Clin Invest 2005; 115: 599-609.

22. Hayashidani S, Tsutsui $\mathrm{H}$, Ikeuchi $\mathrm{M}$, et al. Targeted deletion of MMP-2 attenuates early LV rupture and late remodeling after experimental myocardial infarction. Am J Physiol Heart Circ Physiol 2003; 285: H1229-35.

23. Cogni AL, Farah E, Minicucci MF, et al. Metalloproteinases-2 and -9 predict left ventricular remodeling after myocardial infarction. Arq Bras Cardiol 2013; 100: 315-21.

24. Yurchenko V, Constant S, Bukrinsky M. Dealing with the family: CD147 interactions with cyclophilins. Immunology 2006; 117: 301-9.

25. Seizer P, Schönberger T, Schött M, et al. EMMPRIN and its ligand cyclophilin A regulate MT1-MMP, MMP-9 and M-CSF during foam cell formation. Atherosclerosis 2010; 209: 51-7.

26. Zhang Y, Yip GW, Chan AK, et al. Left ventricular systolic dyssynchrony is a predictor of cardiac remodeling after myocardial infarction. Am Heart J 2008; 156: 1124-32.

27. Nucifora G, Bertini M, Marsan NA, et al. Impact of left ventricular dyssynchrony early on left ventricular function after first acute myocardial infarction. Am J Cardiol 2010; 105: 306-11.

28. Thygesen K, Alpert JS, White HD; Joint ESC/ACCF/AHA/WHF Task Force for the Redefinition of Myocardial Infarction. Universal definition of myocardial infarction. J Am Coll Cardiol 2007; 50: 2173-95.

29. Lang RM, Bierig M, Devereux RB, et al. Chamber Quantification Writing Group; American Society of Echocardiography's Guidelines and Standards Committee; European Association of Echocardiography. Recommendations for chamber quantification: a report from the American Society of Echocardiography's Guidelines and Standards Committee and the Chamber Quantification Writing Group, developed in conjunction with the European Association of Echocardiography, a branch of the European Society of Cardiology. J Am Soc Echocardiogr 2005; 18: 1440-63.

30. Ellis SG, Vandormael MG, Cowley MJ, et al. Coronary morphologic and clinical determinants of procedural outcome with angioplasty for multivessel coronary disease. Implications for patient selection. Multivessel Angioplasty Prognosis Study Group. Circulation 1990; 82: 1193-202.

31. Quiñones MA, Otto CM, Stoddard M, et al. Recommendations for quantification of Doppler echocardiography: a report from the Doppler Quantification Task Force of the Nomenclature and Standards Committee of the American Society of Echocardiography. J Am Soc Echocardiogr 2002; 15: 167-84.

32. Kapetanakis S, Kearney M, Siva A, Gall N, Cooklin M, Monaghan M. Real-time three-dimensional echocardiography: a novel technique to quantify global left ventricular mechanical dyssynchrony. Circulation 2005; 112: 992-1000.

33. The GUSTO Angiographic Investigators. The effects of tissue plasminogen activator, streptokinase, or both on coronary-artery patency, ventricular function, and survival after acute myocardial infarction. New Engl J Med 1993; 329: 1615-22.

34. Gibson CM, Cannon CP, Murphy SA, et al. Relationship of TIMI myocardial perfusion grade to mortality after administration of thrombolytic drugs. Circulation 2000; 101: 125-30.

35. Ellis SG, Vandormael MG, Cowley MJ, et al. Coronary morphologic and clinical determinants of procedural outcome with angioplasty for multivessel coronary disease. Implications for patient selection. Multivessel Angioplasty Prognosis Study Group. Circulation 1990; 82: 1193-202.

36. Piot $\mathrm{C}$, Croisille $\mathrm{P}$, Staat $\mathrm{P}$, et al. Effect of cyclosporine on reperfusion injury in acute myocardial infarction. New Engl J Med 2008; 359: 473-81.

37. Mewton N, Croisille P, Gahide G, et al. Effect of cyclosporine on left ventricular remodeling after reperfused myocardial infarction. J Am Coll Cardiol 2010; $55:$ 1200-5

38. Sheu JJ, Chua S, Sun CK, et al. Intra-coronary administration of cyclosporine limits infarct size, attenuates remodeling and preserves left ventricular function in porcine acute anterior infarction. Int J Cardiol 2011; 147: 79-87.

39. Gill RS, Bigam DL, Cheung PY. The role of cyclosporine in the treatment of myocardial reperfusion injury. Shock 2012; 37: 341-7.

40. Chen Z, Siu B, Ho YS, et al. Overexpression of MnSOD protects against myocardial ischemia/reperfusion injury in transgenic mice. J Mol Cell Cardiol 1998; 30: 2281-9.

41. Wang $\mathrm{P}$, Chen $\mathrm{H}$, Qin $\mathrm{H}$, et al. Overexpression of human copper, zinc-superoxide dismutase (SOD1) prevents postischemic injury. Proc Natl Acad Sci USA 1998; 95: 4556-60.

42. Nakamura K, Fushimi K, Kouchi H, et al. Inhibitory effects of antioxidants on neonatal rat cardiac myocyte hypertrophy induced by tumor necrosis factor-alpha and angiotensin II. Circulation 1998; 98: 794-9.

43. Siwik DA, Chang DL, Colucci WS. Interleukin-1beta and tumor necrosis factor-alpha decrease collagen synthesis and increase matrix metalloproteinase activity in cardiac fibroblasts in vitro. Circ Res 2000; 86: 1259-65.

44. Deten A, Holzl A, Leicht $\mathrm{M}$, et al. Changes in extracellular matrix and in transforming growth factor beta isoforms after coronary artery ligation in rats. J Mol Cell Cardiol 2001; 33: 1191-207.

45. Satoh K, Godo S, Saito H, et al. Dual roles of vascular-derived reactive oxygen species-With a special reference to hydrogen peroxide and cyclophilin A. J Mol Cell Cardiol 2014; 73: 50-6.

46. Yuan $\mathrm{W}, \mathrm{Ge} \mathrm{H}, \mathrm{He}$ B. Pro-inflammatory activities induced by CyPA-EMMPRIN interaction in monocytes. Atherosclerosis 2010; 213: 415-21.
47. Abdulla J, Barlera S, Latini $\mathrm{R}$, et al. A systematic review: effect of angiotensin converting enzyme inhibition on left ventricular volumes and ejection fraction in patients with a myocardial infarction and in patients with left ventricular dysfunction. Eur J Heart Fail 2007; 9: 129-35.

48. Komajda M, Lutiger B, Madeira $\mathrm{H}$, et al. Tolerability of carvedilol and ACE-Inhibition in mild heart failure. Results of CARMEN (Carvedilol ACE-Inhibitor Remodelling Mild CHF EvaluatioN). Eur J Heart Fail 2004; 6: 467-75.

49. Sundell J, Engblom E, Koistinen J, et al. The effects of cardiac resynchronization therapy on left ventricular function, myocardial energetics, and metabolic reserve in patients with dilated cardiomyopathy and heart failure. J Am Coll Cardiol 2004; 43: 1027-33

50. Leclercq C, Kass DA: Retiming the failing heart: Principles and current clinical status of cardiac resynchronization. J Am Coll Cardiol 2002; 39: 194-201.

51. Ko JS, Jeong MH, Lee MG, et al. Left ventricular dyssynchrony after acute myocardial infarction is a powerful indicator of left ventricular remodeling. Korean Circ J 2009; 39: 236-42.

52. Mollema SA, Liem SS, Suffoletto MS, et al. Left ventricular dyssynchrony acutely after myocardial infarction predicts left ventricular remodeling. J Am Coll Cardiol 2007; 50: 1532-40.

53. Shin $\mathrm{SH}$, Hung CL, Uno $\mathrm{H}$, et al; Valsartan in Acute Myocardial Infarction Trial (VALIANT) Investigators. Mechanical dyssynchrony after myocardial infarction in patients with left ventricular dysfunction, heart failure, or both. Circulation 2010; 121: 1096-103.

54. Rohde LE, Ducharme A, Arroyo LH, et al. Matrix metalloproteinase inhibition attenuates early left ventricular enlargement after experimental myocardial infarction in mice. Circulation 1999; 99: 3063-70.

55. Nilsson L, Hallén J, Atar D, et al. Early measurements of plasma matrix metalloproteinase-2 predict infarct size and ventricular dysfunction in ST-elevation myocardial infarction. Heart 2012; 98: 31-6.

56. Zhang Y, Chan AK, Yu CM, et al. Left ventricular systolic asynchrony after acute myocardial infarction in patients with narrow QRS complexes. Am Heart J 2005; 149: 497-503.

57. Mahrholdt H, Wagner A, Holly TA, et al. Reproducibility of chronic infarct size measurement by contrast-enhanced magnetic resonance imaging. Circulation 2002; 106: 2322-7. 